\title{
Quantifying the order of spontaneous ripple patterns on ion-irradiated Si(111)
}

\author{
H. Bola George ${ }^{1}$, Ari-David Brown ${ }^{2 *}$, Matthew R. McGrath ${ }^{3}$, Jonah Erlebacher ${ }^{2}$ \& Michael J. \\ Aziz ${ }^{1}$ \\ ${ }^{1}$ Division of Engineering \& Applied Sciences, Harvard University, Cambridge, MA 02138 \\ ${ }^{2}$ Department of Materials Science \& Engineering, Johns Hopkins University, Baltimore, MD \\ 21218 \\ ${ }^{3}$ Physics \& Astronomy Department, Vanderbilt University, Nashville, TN 37235 \\ * Current address: NASA Goddard Space Flight Center, Greenbelt, MD 20771
}

\begin{abstract}
Uniform keV ion irradiation causes a morphological instability known to result in the spontaneous formation of topographic ripple and dot patterns. The degree of order of these patterns, which has important implications for non-lithographic patterning applications, varies markedly with fabrication conditions. We investigate the influence of systematic variations of fabrication conditions, including current density, ion fluence and ion energy, on the degree of order of argon ion bombarded Si(111) surfaces. For quantifying order in sputter rippled topographic images, we develop an algorithm that evaluates the density of topological defects, such as ripple bifurcations and terminations, and suitably normalizes the result in order to present a scalar figure of merit: the normalized defect density. We discuss fabrication conditions that lead to extremely well ordered dot and ripple patterns upon irradiation.
\end{abstract}

\section{INTRODUCTION}

Sputter patterning employs unfocused low-energy ion beams to sputter etch surfaces and is envisioned to play an important role in nanoscale device fabrication - it offers an alternate, highthroughput means of forming periodic structures possessing nanometer length scales. Following ion irradiation, self-organization of one-dimensional ripple arrays have been observed on metal ${ }^{1-}$ 4 , semiconductor ${ }^{5-7}$, and insulator ${ }^{8,9}$ surfaces. In addition, two-dimensional dot arrays have been shown to form under a variety of ion patterning conditions and substrates. Frost and co-workers 10,11 observed the formation of dots possessing hexagonal and rectangular symmetry during ion bombardment of simultaneously rotating InP substrates. Hexagonal dot arrays have also been observed on Si surfaces following ion sputtering at normal incidence with ${ }^{12-16}$ and without ${ }^{17}$ sample rotation; oblique incidence ion sputtering under sample rotation also results in highly ordered GaSb dot arrays ${ }^{18}$. Recently, we reported ${ }^{19-21}$ the formation of sub-micron sized dots possessing quasi-rectangular symmetry resulting from oblique incidence ion bombardment of heated $\mathrm{Si}(111)$ surfaces.

A drawback of the sputter patterning technique pertains to the regularity and order of structure. While short-range order (SRO) of these dots can be quite high, the dots are often separated by defects resulting in dots arrays possessing poor long-range order (LRO) envisaged for some applications. Generally ${ }^{10,12,18,22}$, it has been observed that enhanced dot order is proportional to the ion fluence (sputtering time). The effect of substrate temperature has also been shown to have an impact on dot organization in some instances ${ }^{11}$ - temperature differences of $<50 \mathrm{~K}$ were sufficient to change both the order and the short-range feature symmetry. Recently, the incidence ion angle and beam divergence have been observed to play a role in dot 
formation ${ }^{17,23}$. Theoretical approaches via nonlinear continuum equations for dot evolution on ion bombarded surfaces, e.g. [24], have been applied to GaSb and InP surfaces; dot ordering/defect annihilation is predicted to depend on the relative strengths of nonlinear terms. Recently, we developed a method of quantifying pattern regularity from atomic force microscope (AFM) topographs and reported the normalized density $N_{D}$ of “dislocation-like” defects as a scalar measure of ripple order in $\mathrm{Si}(001)$ samples evolving under the effect of lithographically patterned templates ${ }^{25}$.

In this work, the variation of $N_{D}$ with experimentally controllable parameters, including substrate temperature, ion energy, ion fluence, and ion flux, is presented for oblique incidence $\mathrm{Ar}^{+}$sputter patterned $\mathrm{Si}(111)$ surfaces. We demonstrate that $N_{D}$ is a peaked function of one of these experimentally controllable parameters and that the peak position correlates well with the formation of structures with quasi-rectangular symmetry. Furthermore, we determine the value of $N_{D}$ corresponding to maximum defect density, allowing us to identify and predict directions in which to move to reduce defect density.

\section{EXPERIMENTAL DETAILS}

The ion sputtering experiments of $\operatorname{Si}(111)$ (n-type, As-doped, 0.002-0.020 $\Omega \mathrm{cm}$ ) wafers were carried out in an UHV chamber with base pressure of $6.0 \times 10^{-11}$ Torr. In all instances reported here, a Kaufmann-type RF ion source produced a collimated beam of $\mathrm{Ar}^{+}$ions directed at an incidence angle of $60^{\circ}$ from the sample normal. Other parameters including ion energy, flux and substrate temperature, were varied between 250 and $1200 \mathrm{eV}, 0.38$ and $3.0 \mathrm{~mA} / \mathrm{cm}^{2}$, and 637 and $717^{\circ} \mathrm{C}$, respectively. The substrate temperature was monitored via a spring-loaded thermocouple in contact with the back of the sample and an infrared thermometer that probed the sample surface; the feedback temperature control employed a pyrolytic boron nitride/graphite backside radiant heater. We sputtered the samples for different amounts of time, ranging between 10 and $960 \mathrm{~min}$. The sputtered surface morphologies were analyzed with an atomic force microscope (AFM) in contact mode. The algorithm for calculating $N_{D}$, described elsewhere ${ }^{25}$, was applied to the AFM topographs to determine order as a function of various parameters. Relevant details from the algorithm include: (a) flattening a grayscale AFM topograph by subtracting a quadratic background in $x$ and $y$, (b) reducing the topograph to a binary image using a threshold, obtained via Otsu's method ${ }^{26}$, (c) thinning the white regions to single-pixel wide lines; (d) counting the number of defects identified as the number of junctions or line segment ends in the thinned image, and (e) normalization by multiplying the number of defects by the feature wavelength squared divided by the image area. An AFM topograph, its thresholded binary and thinned representations are shown in Figure 1 (a) through (c).

(a)

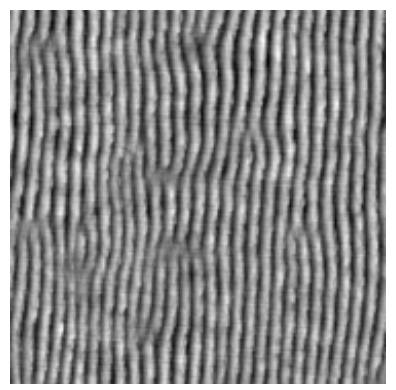

(b)

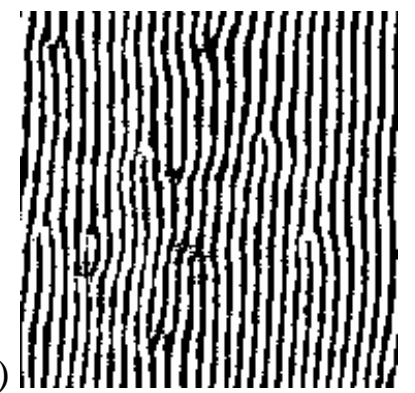

(c)

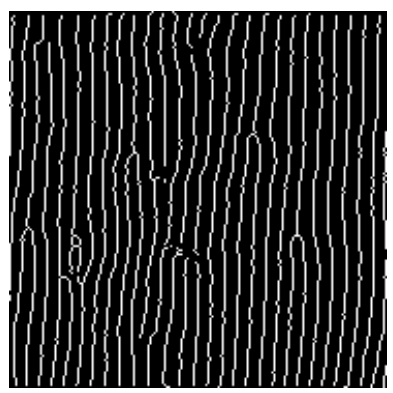

Figure 1: $10 \times 10 \mu^{2} \mathrm{Si}(111)$ sample under $717^{\circ} \mathrm{C}, 750 \mathrm{eV} \mathrm{Ar}{ }^{+}, 256 \mathrm{mins}, 0.75 \mathrm{~mA} \mathrm{~cm}^{-2}, 60^{\circ}$ from normal irradiation. (a) AFM topograph, (b) thresholded image, and (c) thinned version. 


\section{RESULTS \& DISCUSSION}

We have established that the fluence (and not the flux or time) is the control parameter - in a series of constant-fluence runs with different fluxes, we did not observe any systematic variation of $N_{D}$ or the roughness with flux.

We investigated the effect of increasing fluence on surface topography in two ways. In the first, fluence variation was achieved with constant flux and different sputtering times; images from samples sputtered under nominally identical conditions for different times are shown below, Figure 2a-c. In the second case, the irradiation time was fixed while samples were sputtered with different fluxes; representative images are shown below (Figure $2 \mathrm{~d}-\mathrm{f}$ ) For samples sputtered under similar conditions, we show AFM topographs at both the low- (Figure 2a and d) and high-fluence (Figure 2c and f) regimes where we observe parallel mode (i.e., ripple wave vector parallel to the projected beam) and perpendicular mode (i.e., ripple wave vector perpendicular to projected beam) ripples, respectively. For intermediate values between both low- and high-fluence limits, the AFM images show the parallel mode ripples being chopped up by the developing perpendicular mode ripples. For example, in Figure 2e, the AFM image at an intermediate fluence shows the truncated structures resulting from superposition of ripple periodicities in both orthogonal directions. Thus, we observe the transition through three different morphology regimes on ion-irradiated $\mathrm{Si}(111)$ as fluence increases.

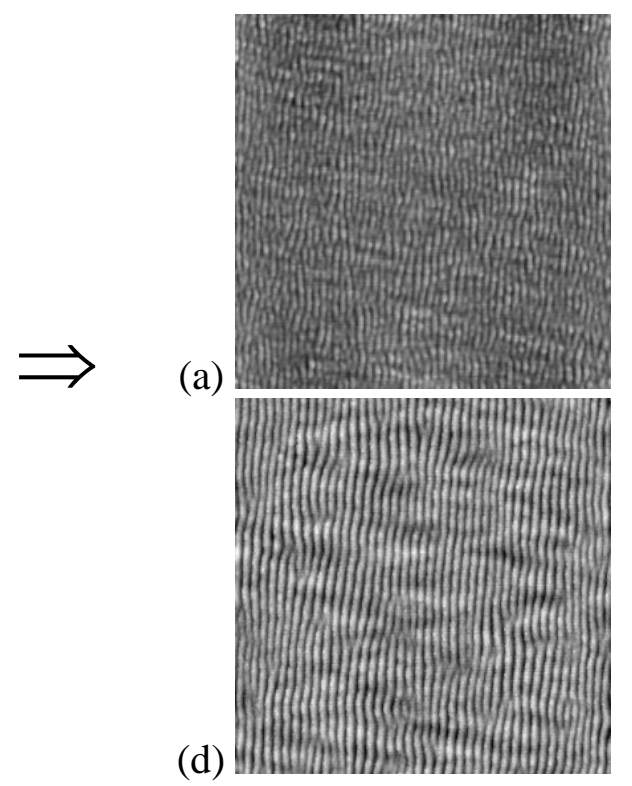

(b)

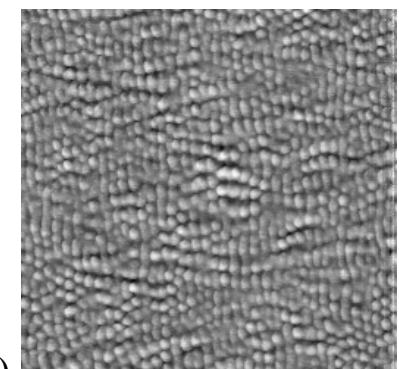

(e)

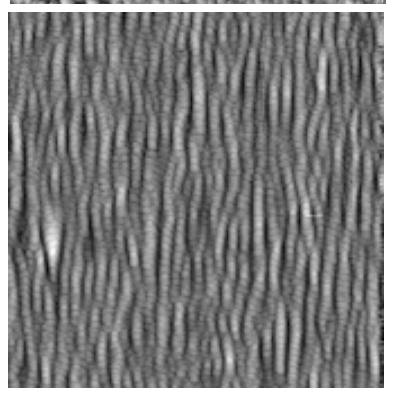

(c)

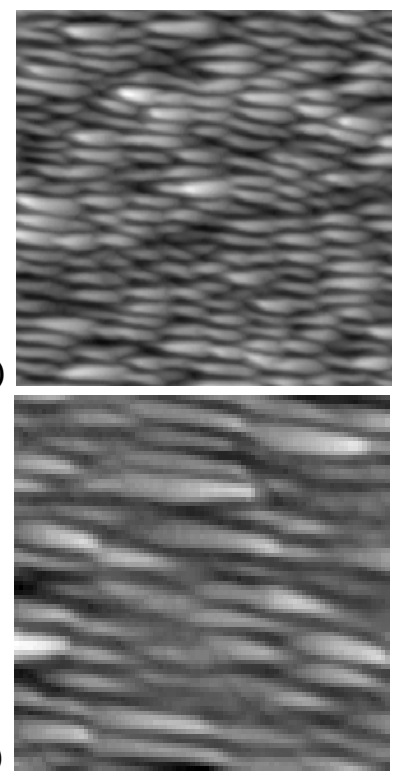

Figure 2: $(\mathrm{a}-\mathrm{c})$ Evolution of topographic pattern with increasing fluence at constant flux. Samples were sputtered for 20, 80, and 171 minutes at $657^{\circ} \mathrm{C}, 60^{\circ}$ from normal, $0.75 \mathrm{~mA} \mathrm{~cm}{ }^{-2} \mathrm{Ar}^{+}$ions (10 mA beam) for (a), (b), and (c), respectively; images are $15 \times 15 \mu \mathrm{m}^{2}$. Parallel and perpendicular mode ripples are dominant in (a) and (c), respectively. (d-f) Evolution under constant time with varying fluxes; images used were sputtered for 256 mins with $500 \mathrm{eV} \mathrm{Ar}^{+}$ions at $717{ }^{\circ} \mathrm{C}, 60^{\circ}$ from normal. (d) $20 \times 20 \mu \mathrm{m}^{2}$ AFM topograph at $0.75 \mathrm{~mA} \mathrm{~cm}{ }^{-2} \mathrm{Ar}^{+}$ions (10 mA beam), (e) $35 \times 35 \mu \mathrm{m}^{2}$ AFM topograph at $1.65 \mathrm{~mA}$ $\mathrm{cm}^{-2} \mathrm{Ar}^{+}$ions (22 mA beam), and (f) $15 \times 15 \mu \mathrm{m}^{2}$ AFM topograph at $2.48 \mathrm{~mA} \mathrm{~cm} \mathrm{Ar}^{+}$ions (33 mA beam); the ripples in (d) and (f) are parallel and perpendicular mode, respectively. The horizontal arrow indicates the projected beam direction. 
In Figure 3a, we show the effect of fluence on $N_{D}$. We observe that the normalized defect density is at a minimum at low- and high-fluence regimes where single-mode ripples are dominant, increases and reaches a peak value at intermediate fluences, and decreases again as the fluence increases. In these experiments, the samples were sputtered at two different temperatures, 690 and $717^{\circ} \mathrm{C}$, under identical conditions (500 eV Ar${ }^{+}, 60^{\circ}$ and $\left.256 \mathrm{~min}\right)$. To effect fluence variations, the sputtering time was kept constant (256 min) while the flux was adjusted. Qualitatively, we observe similar behavior under nominally identical conditions at both 690 and $717^{\circ} \mathrm{C}$, although the peak in the $N_{D}$ versus fluence curve is shifted to a lower fluence value $\left(6.5 \times 10^{19} \mathrm{Ar}^{+} \mathrm{cm}^{-2}\right)$. The surface roughness of the sputtered samples, measured from AFM images, increases monotonically with fluence, see Figure $3 \mathrm{~b}$.

The early-rise of the peak in $N_{D}$ versus fluence plot (Figure 3a) at both temperatures can be explained based on our observations of a strong correlation between the onset of ripple formation and the substrate temperature, i.e., the higher the sample temperature, the longer it takes before ripples appear. The earlier onset is also consistent with the Bradley-Harper (BH) linear instability theory ${ }^{27}$; at higher temperatures, the amplification rate of the instability is reduced. However, no existing theory makes any predictions for the ripple mode transition from parallel to perpendicular under fluence evolution.

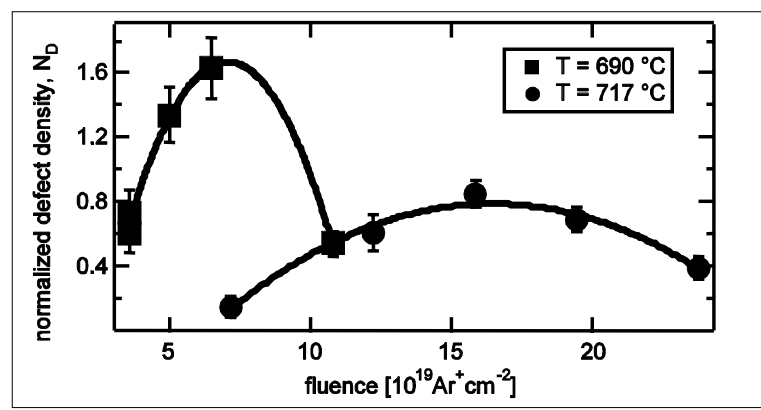

(a)

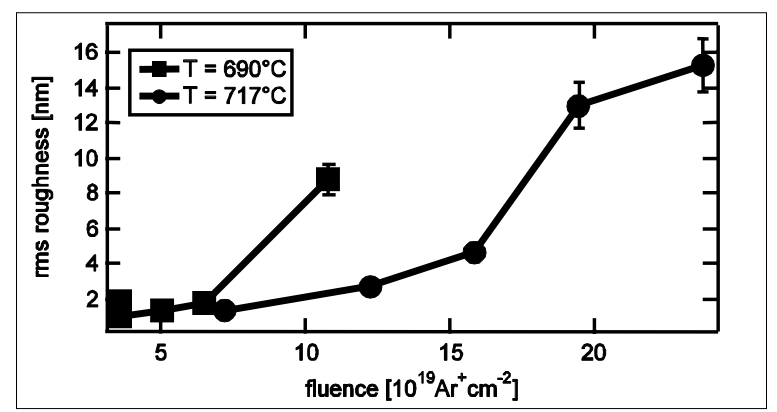

(b)

Figure 3: (a) Normalized defect density and (b) surface roughness, respectively, versus fluence at 690 (squares) and $717^{\circ} \mathrm{C}$ (circles), constant time $=256 \mathrm{~min}, 500 \mathrm{eV} \mathrm{Ar}^{+}, 60^{\circ}$ from normal; the lines are drawn as guides to the eye. Vertical error bars in (a) based on the uncertainty in the measured wavelength; in (b), the errors bars are $10 \%$ of the base values.

We also investigated the effect of ion energy on the regularity of surface ripples. Generally, calculated $N_{D}$ values reflect the trend we observe - higher energies lead to better ordered ripples, i.e. fewer dislocation-type defects. A series of images is shown in Figure 4(a) through (d) sputtered under nominally identical conditions $\left(717^{\circ} \mathrm{C}, 0.75 \mathrm{~mA} / \mathrm{cm}^{2}\right.$ and $256 \mathrm{~min}$ ) at different energies. $N_{D}$ and rms roughness are shown in Figure 4 (e) and (f). The degree of order of the ripples increases with energy; the rms roughness is virtually constant except at the lowest energy.

This effect of the energy on the ripple order is also independently confirmed by monitoring the width of the surface power spectrum from in-situ light scattering spectroscopy (LiSSp); as the ion energy decreases, the LiSSp peak becomes considerably broader. $N_{D}$ is a measure of the geometric defect density whereas the FFT is a measure of the surface periodicity. Thus, calculation of $N_{D}$ is a more useful parameter to characterize samples with low defect densities; such samples would only be expected to have an effect on the FFT in the low frequency, high noise region of the spectrum. Additionally, in cases where samples have pre-patterned 
boundaries, $N_{D}$ is much more useful than FFT-based algorithms since FFT higher harmonics of the boundaries obscures the contribution of the rippled regions ${ }^{25}$.

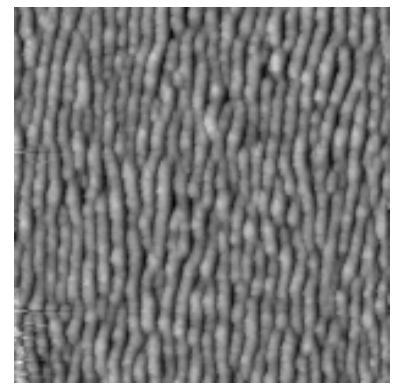

(a)

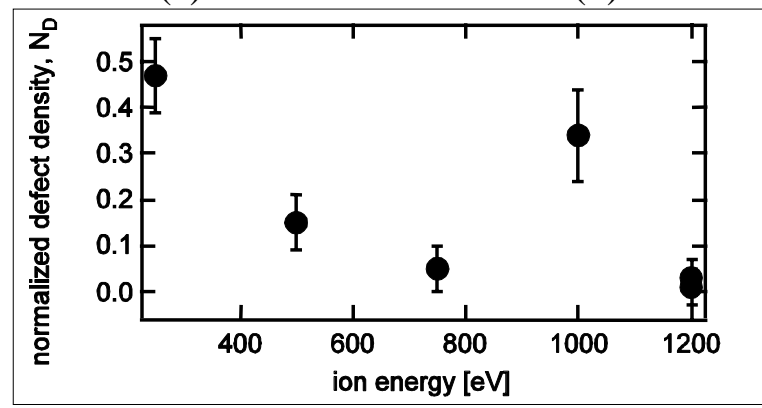

(e)

(b)

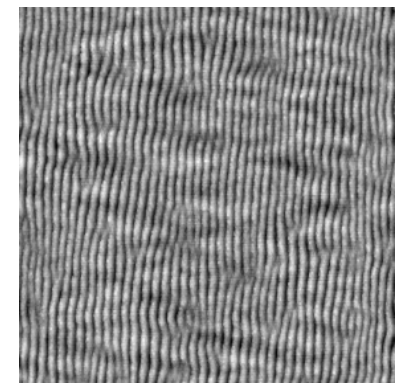

Figure 4: Energy dependence of surface pattern at $717^{\circ} \mathrm{C}, 256 \mathrm{mins}, 0.75 \mathrm{~mA} \mathrm{~cm}{ }^{-2} \mathrm{Ar}^{+}$ions $(10 \mathrm{~mA}$ beam), and 60-degree incident beam. (a), (b), and (c) $20 \times 20 \mu \mathrm{m}^{2}$ AFM topographs sputtered at 250, 500, and $1000 \mathrm{eV}$, respectively. (d) $10 \times 10 \mu \mathrm{m}^{2}$ topograph following sputtering at $1200 \mathrm{eV}$. (e) Normalized defect density vs. ion energy and (f) the surface roughness vs. ion energy.

The ripples under the conditions of Figure 4 are of the parallel mode. At $1000 \mathrm{eV}$, it appears both ripple modes are present (Figure 4c) whereas at the next lowest and highest energies, 500 (Figure 4b) and $1200 \mathrm{eV}$ (Figure 4d), respectively, single-mode ripples persist. We are not sure why both modes are pronounced at $1000 \mathrm{eV}$; while the ripples are contiguous to the eye, the pattern developing in the orthogonal direction effectively chops up the ripples into patches following thresholding, thereby resulting in an abnormally high value for $N_{D}$. We do not rule out the role of beam divergence having an effect on surface morphology; we observe that our beam becomes more collimated at higher energies. Changes in the beam collimation have been associated with changes in surface topography ${ }^{23}$.

\section{SUMMARY}

Systematic variations of fabrication conditions, including current density, ion fluence and ion energy, influence the degree of order of argon ion bombarded Si(111) surfaces. Our algorithm for calculating the normalized defect density can be used to quantify the morphological surface changes during sputtering. As the fluence increases in samples sputtered under nominally identical conditions, parallel-mode ripples initially form; however, upon further irradiation, the parallel-mode ripples are washed out as the perpendicular-mode ripples develop, $N_{D}$ is maximized in the middle of this transition. Higher energies tend to lead to more ordered structures. 


\section{ACKNOWLEDGMENTS}

Research at Johns Hopkins University was supported by DE-FG02-01ER45942. Research at Harvard University was supported initially by DE-FG02-01ER45947 and subsequently by

NSF- DMR-0306997.

\section{REFERENCES}

P. Haymann, Comptes Rendus Hebdomadaires des Seances de l'Academie des Sciences 248 (17), 2472 (1959).

S. Rusponi, G. Costantini, C. Boragno et al., Phys. Rev. Lett. 81 (13), 2735 (1998).

S. Rusponi, G. Costantini, C. Boragno et al., Phys. Rev. Lett. 81 (19), 4184 (1998).

W.-L. Chan, N. Pavenayotin, and E. Chason, Phys. Rev. B 69 (24), 245413 (2004).

E. Chason, T.M. Mayer, B.K. Kellerman et al., Phys. Rev. Lett. 72 (19), 3040 (1994).

G. Carter and V. Vishnyakov, Phys. Rev. B 54 (24), 17647 (1996).

J. Erlebacher, M.J. Aziz, E. Chason et al., Phys. Rev. Lett. 82 (11), 2330 (1999).

T.M. Mayer, E. Chason, and A.J. Howard, J. Appl. Phys. 76, 1633 (1994).

C. C. Umbach, R. L. Headrick, and K. C. Chang, Phys. Rev. Lett. 87 (24), 246104 (2001).

F. Frost, A. Schindler, and F. Bigl, Phys. Rev. Lett. 85 (19), 4116 (2000).

F. Frost and B. Rauschenbach, Appl. Phys. A 77 (1), 1 (2003).

R. Gago, L. Vazquez, R. Cuerno et al., Appl. Phys. Lett. 78 (21), 3316 (2001).

A. Cuenat and M.J. Aziz, Mater. Res. Soc. Symp. Proc. 696, N2.8.1 (2002).

F. Ludwig, C.R. Eddy, O. Malis et al., Appl. Phys. Lett. 81 (15), 2770 (2002).

L.J. Qi, W.Q. Li, X.J. Yang et al., Chinese Physics Letters 22 (2), 431 (2005).

L.J. Qi, L. Li, W.Q. Li et al., Chinese Physics 14 (8), 1626 (2005).

B. Ziberi, F. Frost, B. Rauschenbach et al., Appl. Phys. Lett. 87 (3), 033113 (2005).

S. Facsko, T. Dekorsy, C. Koerdt et al., Science 285 (5433), 1551 (1999).

A.-D. Brown, H.B. George, M.J. Aziz et al., Mat. Res. Soc. Symp. Proc. R.7.8, 792 (2004).

A.-D. Brown, J. Erlebacher, W.-L. Chan et al., Phys. Rev. Lett. 95 (5), 056101 (2005).

A.-D. Brown and J. Erlebacher, Phys. Rev. B 72, 075350 (2005).

T. Bobek, S. Facsko, T. Dekorsy et al., Nucl. Instrum. \& Meth. B 178, 101 (2001).

B. Ziberi, F. Frost, M. Tartz et al., Thin Solid Films 459, 106 (2004).

M. Castro, R. Cuerno, L. Vazquez et al., Phys. Rev. Lett. 94 (1), 016102 (2005).

A. Cuenat, H.B. George, K.-C. Chang et al., Adv. Mat. 17, 2845 (2005).

N. Otsu, IEEE Transactions on Systems Man and Cybernetics 9 (1), 62 (1979).

R.M. Bradley and J.M. Harper, J. Vac. Sci. Technol. A 6, 2390 (1988). 